\title{
Overexpression of miR-148a inhibits viability and invasion of ovarian cancer OVCAR3 cells by targeting FOXO3
}

\author{
DANDAN ZHU ${ }^{1 *}$, DONGLAN YUAN ${ }^{1 *}$, RUNFA GUO $^{1}$, LIXIN ZHANG $^{1}$, TING GUO $^{2}$, \\ YINLING ZHAO $^{1}$, JIA WANG ${ }^{1}$, XINPING CHEN ${ }^{1}$, HUA QIAN $^{1}$ and HONGSHAN GE ${ }^{1}$ \\ ${ }^{1}$ Department of Obstetrics and Gynecology; ${ }^{2}$ Central Laboratory, Taizhou People's Hospital, \\ Fifth Affiliated Hospital to Nantong University, Taizhou, Jiangsu 225300, P.R. China
}

Received May 18, 2018; Accepted March 29, 2019

DOI: $10.3892 / \mathrm{ol} .2019 .10321$

\begin{abstract}
Decreased expression of microRNA (miR)-148a is associated with poor prognosis in ovarian cancer. The aim of the present study was to investigate the impact of miR-148a on tumor cell viability and invasion via targeting forkhead box protein O3 (FOXO3). Expression of miR-148a was detected in paired tumor and adjacent normal tissues. OVCAR3 cells were transfected with miR-148a mimic and inhibitor. Cell viability, apoptosis and invasion were determined. A luciferase reporter assay was used to study the association between miR-148a and FOXO3. In addition, the influence of miR-148a on tumor cell growth was investigated by performing xenograft assays in nude mice. RT-qPCR showed that miR-148a was downregulated in ovarian cancer tissues. Overexpression of miR-148a in OVCAR3 cells inhibited cell viability, suppressed invasion and promoted cellular apoptosis. The dual-luciferase assay indicated that miR-148a directly regulated the expression of FOXO3, a transcription factor of caspase-3. Western blotting confirmed that the expression of caspase- 3 was regulated by the modulation of miR-148a expression. In vivo assays revealed that miR-148a overexpression inhibited the growth of OVCAR3 xenograft tumors in nude mice. miR-148a is a tumor suppressor in ovarian cancer OVCAR3 cells and in nude mice. The suppressive effect is due to inhibiting cell viability and invasion as well as promoting apoptosis. These results may provide theoretical
\end{abstract}

Correspondence to: Dr Hua Qian or Dr Hongshan Ge, Department of Obstetrics and Gynecology, Taizhou People's Hospital, Fifth Affiliated Hospital to Nantong University, 399 Hailing South Road, Taizhou, Jiangsu 225300, P.R. China

E-mail: qhtzry@outlook.com

E-mail: dafeng76@126.com

${ }^{*}$ Contributed equally

Key words: forkhead box protein O3, invasion, microRNA-148a, ovarian cancer, cell viability basis for targeting miR-148a in the treatment of ovarian cancer.

\section{Introduction}

Ovarian cancer is a common solid tumor in women and, as reported by the National Central Cancer Registry of China, there were $\sim 521,000$ new cases and 225,000 deaths in 2015 (1). The increasing trend in mortality rate of ovarian cancer may be due to disease recurrence, despite significant advances in early detection and therapy $(2,3)$. To improve treatment efficacy and reduce recurrence, a better understanding of the etiology of ovarian cancer is urgently required.

MicroRNAs (miRNAs/miR) are a class of small non-coding RNAs that are widely involved in the occurrence of cancer (4). They have emerged as useful biomarkers for early diagnosis and prognosis as these small molecules are stable in blood and highly tumor-specific (5). A recent systematic review of observational studies investigated the prognostic significance of miR-148a in various cancers and reported a correlation between low miR-148a levels and poor overall survival in patients with cancer (6). miR-148a has been found to be dysregulated in several cancers, including ovarian cancer $(7)$, breast cancer $(8,9)$, colorectal cancer $(10)$, gastrointestinal cancer (11), prostate cancer (12), hepatocellular carcinoma (13), papillary thyroid carcinoma (14) and renal cancer (15). In ovarian cancer, miR-148a is decreased in the plasma of patients compared with healthy controls and its expression level is positively associated with survival time (16). In vitro cellular experiments have demonstrated that miR-148a inhibits proliferation, migration and invasion of ovarian cancer cell lines SKOV-3, OVCAR, ES-2 and A2780 (7,16,17).

At present, the genes targeted by miR-148a are not fully elucidated. Forkhead box protein O3 (FOXO3, also known as FKHRL1) is predicted to be a target of miR-148a. FOXO3 belongs to the $\mathrm{O}$ subfamily of FOX transcription factors (18). The FOXO factors regulate activities of multiple signaling pathways and serve critical roles during cellular growth, survival and carcinogenesis (19). Therefore, it was hypothesized that miR-148a may act as a tumor suppressor in ovarian cancer by regulating FOXO3 expression. In vitro and in vivo experiments were performed to test this hypothesis. The present study may provide novel evidence for the treatment of ovarian cancer. 


\section{Materials and methods}

Reagents. DMEM cell culture media (Gibco; Thermo Fisher Scientific, Inc.) supplemented with 10\% FBS (Gibco; Thermo Fisher Scientific, Inc.) was used for cell studies. Lipofectamine 2000 transfection kit and the SYBR Green PCR Master mix qPCR kit were purchased from Invitrogen (Thermo Fisher Scientific, Inc.). Oligonucleotides used for transfection and primers used for RT-qPCR were synthesized by Shanghai GenePharma Co., Ltd. The Cell Counting kit-8 (CCK-8), the apoptosis assay kit, RIPA lysis buffer and the enhanced chemiluminescence (ECL) kit were purchased from Beyotime Institute of Biotechnology. The Dual-Luciferase Assay kit was purchased from Promega Corporation. The RNAiso Plus RNA extraction kit and PrimeScript 1st strand cDNA Synthesis kit were purchased from Takara Biotechnology Co., Ltd. The antibodies used for western blotting were purchased from Abcam.

Tissue samples and cell culture. A total of 20 pairs of ovarian epithelial cancer tissues and matched normal adjacent tissues were obtained from 20 female patients (median age, 54 years; range,39-76 years) who received surgical resection at the Jiangsu Taizhou People's Hospital (Taizhou, China). The samples were collected between January 2013 and March 2017. Diagnosis was based on histopathological evaluation of hematoxylin and eosin-stained tissue samples. Histological classification was determined based on the World Health Organization Histological Classification criteria (20). The cancer stage of the samples was determined according to the International Federation of Gynecology and Obstetrics criteria (21). The criteria were: i) Tumor was confined to ovaries or fallopian tube; ii) tumor involved 1 or both ovaries or fallopian tubes with pelvic extension (below pelvic brim) or primary peritoneal cancer; iii) tumor involved 1 or both ovaries or fallopian tubes, or primary peritoneal cancer, with cytologically or histologically confirmed spread to the peritoneum outside the pelvis and/or metastasis to the retroperitoneal lymph node; and iv) distant metastasis excluding peritoneal metastases. Fresh tissues were snap-frozen in liquid nitrogen and stored at $-80^{\circ} \mathrm{C}$. The study protocol was approved by the Ethics Committee Board of Taizhou People's Hospital. Informed consent was obtained from the patients. The clinical information of the patients is shown in Table I. The median value for the relative expression of miR-148a was 1.56 , so the patients were divided into 2 groups based on the relative expression of miR-148a: High miR-148a, >1.56; and low miR-148a, $\leq 1.56$.

Human ovarian cancer cell lines, including ovarian serous adenocarcinoma cell line OVCAR3, ovarian serous cystadenocarcinoma cell line SKOV3 (American Type Culture Collection) and normal human ovarian surface epithelial cells (HOSEpiC; cat. no 7310; ScienCell Research Laboratories, Inc.) were used in the current study. The cells were cultured in DMEM supplemented with $10 \% \mathrm{FBS}$ and kept at $37^{\circ} \mathrm{C}$ in a humidified incubator with $5 \% \mathrm{CO}_{2}$. HOSEpiC cells used for the following experiments were the second passage.

Transfection. All the transfections were done using Lipofectamine 2000 transfection reagent according to the manufacturer's instructions. OVCAR3 and SKOV3 cells were seeded in 6 -well plates $\left(\sim 1 \times 10^{5}\right.$ cells/well $)$ and grown to $70 \%$ confluence. For each well, the cells were transfected with 100 pmol miR-148a mimic (sense, 5'-UCAGUGCAU GACAGA ACU UGG-3' and antisense, 5'-AAGTTCUGU CAUGCACUGAUU-3') or miR-148a inhibitor (5'-ACAAAG UUCUGUAGUGCACUGA-3'). Scrambled miRNA was used as a negative control (sense, 5'-UUCUCCGAACGU GUCACGUTT-3' and antisense, 5'-ACGUGACACGUUCGG AGAATT-3'). The miR-148a mimic and scrambled negative control were designed as previously described (7). Cells were collected $48 \mathrm{~h}$ after transfection, and the transfection efficiency was determined by RT-qPCR.

Cell viability. For the cell viability assay, OVCAR3 and SKOV3 cells were transfected with miR-148a mimic, miR-148a inhibitor or scrambled control for $48 \mathrm{~h}$. Cells $\left(\sim 1 \times 10^{5}\right)$ in $200 \mu \mathrm{l}$ media were added to 96 -well plates. After 24,48 and $72 \mathrm{~h}$, the number of viable cells was measured using CCK- 8 according to manufacturer's protocol. The cell density was measured at $450 \mathrm{~nm}$ on a Bio-Rad Benchmark microplate reader (Bio-Rad Laboratories, Inc.).

Transwell assay. Cellular invasive ability was measured using a Transwell assay. OVCAR3 and SKOV3 cells were transfected with miR-148a mimic, inhibitor or scrambled negative control for $48 \mathrm{~h}$. Cells were then harvested, suspended in serum-free media and seeded in Matrigel-coated 24-well plates (EMD Millipore). For each sample, $2 \times 10^{4}$ cells suspended in $200 \mu \mathrm{l}$ serum-free media were added in the upper chambers and DMEM with $10 \%$ FBS was added in the lower chambers. After incubation for $24 \mathrm{~h}$, non-invasive cells were removed and cells on lower surface were stained with $0.1 \%$ crystal violet at room temperature for $20 \mathrm{~min}$. A total of six random visual fields in each well were visualized under a light microscope for cell counting at $\mathrm{x} 200$ magnification.

Cell apoptosis. For the cell apoptosis assay, OVCAR3 and SKOV3 cells were transfected with miR-148a mimic, inhibitor or scrambled control for $48 \mathrm{~h}$. Cells were then harvested, washed with cold PBS and stained at $4^{\circ} \mathrm{C}$ with Annexin V-FITC and propidium iodide for $10 \mathrm{~min}$. The apoptosis rate ( $10^{5}$ cells/sample) was detected on CytoFlex flow cytometer (Beckman Coulter, Inc.). FlowJo software (version 7.0; FlowJo LLC) was used to analyze flow cytometry data.

Bioinformatics. The prediction of the 3'-UTR of FOXO3 as the binding target of miR-148a binding target was performed using TargetScan software (http://www.targetscan.org). TargetScan is an online bioinformatic tool that predicts the target genes of miRNAs. If the miRNA and gene has paired sequences at the 3'UTR region, the gene was then predicted as a target of the miRNA.

Dual-luciferase reporter assay. The direct targeting interaction between miR-148a and FOXO3 was detected by dual-luciferase reporter assay. The hsa-miR-148a seed sequence in the 3'-untranslated region (UTR) of FOXO3 mRNA was cloned into the pmirGLO vector (Promega Corporation). A mutated sequence was generated and cloned into the vector. The reporter plasmids [FOXO3-wild type (WT) and FOXO3-mutant (MT)] were constructed by Shanghai GenePharma Co., Ltd. OVCAR3 
Table I. Association between miR-148a and clinicopathological parameters of patients with ovarian cancer.

\begin{tabular}{|c|c|c|c|c|}
\hline Parameters & Cases, $\mathrm{n}$ & High miR-148, n & Low miR-148, n & P-value \\
\hline Age, years & & & & 0.1990 \\
\hline$<50$ & 8 & 5 & 3 & \\
\hline$>50$ & 12 & 4 & 8 & \\
\hline Tumor volume, $\mathrm{cm}$ & & & & 0.0072 \\
\hline$<4$ & 13 & 3 & 10 & \\
\hline$>4$ & 7 & 6 & 1 & \\
\hline Histological grade & & & & 0.3428 \\
\hline Well-intermediately & 9 & 3 & 6 & \\
\hline Poor & 11 & 6 & 5 & \\
\hline Distant metastasis & & & & 0.0813 \\
\hline M0 & 13 & 4 & 9 & \\
\hline M1 & 7 & 5 & 2 & \\
\hline Stage & & & & 0.0077 \\
\hline I-II & 11 & 2 & 9 & \\
\hline III-IV & 9 & 7 & 2 & \\
\hline
\end{tabular}

Data were compared using $\chi^{2}$ test. miR, microRNA.

and SKOV3 cells $\left(1 \times 10^{5}\right.$ cells/well) were co-transfected with pmirGLO reporter vector (600 ng) and a control Renilla luciferase vector pRL-CMV (400 ng; Promega Corporation) in the presence of the miR-148a mimic $(50 \mathrm{ng} / \mathrm{ml})$ or scrambled control. After incubation for $48 \mathrm{~h}$, cells were harvested and the luciferase activities were detected using Dual-Luciferase Assay kit. Renilla luciferase activity was used as a reference for normalization

Xenograft of nude mice. OVCAR3 cells were transfected with the miR-148a mimic or inhibitor for $48 \mathrm{~h}$ and harvested for the xenograft assay in nude mice. A total of 36 male C57BL/6 nude mice (weight, 22-24 g; age, 8 weeks; Shanghai SLAC Laboratory Animal Co., Ltd.) were randomly arranged into 3 groups (12/group). Mice were housed at $20-26^{\circ} \mathrm{C}$ with $55 \pm 5 \%$ humidity, $12 \mathrm{~h}$ light/dark cycle and ad libitum access to food and water. Each mouse was injected with $5 \times 10^{6}$ cells (suspended in $100 \mu \mathrm{l}$ PBS) in the lower back subcutaneously. At week 1, 2, 3 and 4 after inoculation, 3 mice were selected from each group and sacrificed to check tumor formation. Mice were sacrificed by intraperitoneal injection of sodium pentobarbital (150-200 mg/kg) Tumor volumes and weight were measured. The tumor volume was calculated with the formula $0.5 \mathrm{x}$ length $\mathrm{x}$ width ${ }^{2}$. Animal health and behavior were monitored every day. None of the animals presented with more than one tumor. The animal experimental protocol was reviewed and approved by the Animal Care and Use Committee of Jiangsu Taizhou People's Hospital.

$R T-q P C R$. Total RNA was extracted from tissue samples and cells using RNAiso Plus kit. First-strand cDNA was synthesized with PrimeScript 1st strand cDNA Synthesis kit (Takara Biotechnology Co., Ltd.) at $37^{\circ} \mathrm{C}$ for $15 \mathrm{~min}$ followed by $85^{\circ} \mathrm{C}$ for $5 \mathrm{sec}$. The expression of miR-148a was quantified by qPCR in $20 \mu \mathrm{l}$ reactions using SYBR Green PCR Master mix. The
PCR reactions were carried out on Applied Biosystems 7500 Real-Time PCR System (Thermo Fisher Scientific, Inc.). The PCR conditions were set as: $95^{\circ} \mathrm{C}$ for $5 \mathrm{~min}$; 40 cycles of $95^{\circ} \mathrm{C}$ for $15 \mathrm{sec}, 60^{\circ} \mathrm{C}$ for $1 \mathrm{~min}$ and $72^{\circ} \mathrm{C}$ for $30 \mathrm{sec}$. The primers used for qPCR were as follows: miR-148a forward, 5'-TCAGTG CACTACAGAACTTTGT-3' and reverse, 5'-GCTGTCAAC GATACGCTACGT-3'; and U6 forward, 5'-CTCGCTTCG GCAGCACA-3' and reverse, 5'-AACGCTTCACGAATTTGC GT-3'. The relative expression of miR-148a was normalized to the expression of U6 using the $2^{-\Delta \Delta \mathrm{Cq}}$ method (22).

Western blotting. Total protein extracts were prepared by lysing tissue and cells in RIPA lysis buffer (Beyotime Institute of Biotechnology). Protein concentrations were determined by bicinchoninic acid protein assay. Equal amounts of proteins $(50 \mu \mathrm{g})$ were subjected to SDS-PAGE separation on $10 \%$ gels, transferred to polyvinylidene difluoride membranes (Merck $\mathrm{KGaA}$ ) and blocked with 5\% skim milk at room temperature for $4 \mathrm{~h}$. Membranes were probed with primary antibodies overnight at $4^{\circ} \mathrm{C}$. The primary antibodies were used at 1:1,000 dilutions as follows: Rabbit monoclonal anti-FOXO3A (cat. no. ab109629), rabbit polyclonal anti-active caspase3 (cat. no. ab2302), rabbit monoclonal anti-pro-Caspase 3 (cat. no. ab32150) and rabbit polyclonal anti-GAPDH (cat. no. ab9485). GAPDH was used as an internal control. The membranes were then probed with goat anti-rabbit $\operatorname{IgG}(1: 2,000$; horseradish peroxidase-conjugated; cat. no. ab205718) at room temperature for $1 \mathrm{~h}$. The chemiluminescence signals were detected by the ECL method. The relative protein expression levels were analyzed with Image-Pro Plus 6.0 software (Media Cybernetics Inc.).

Statistical analysis. The statistical analysis was performed using SPSS 19.0 software (IBM Corp). Continuous variables were expressed as the mean \pm standard deviation. At least three 
A



C

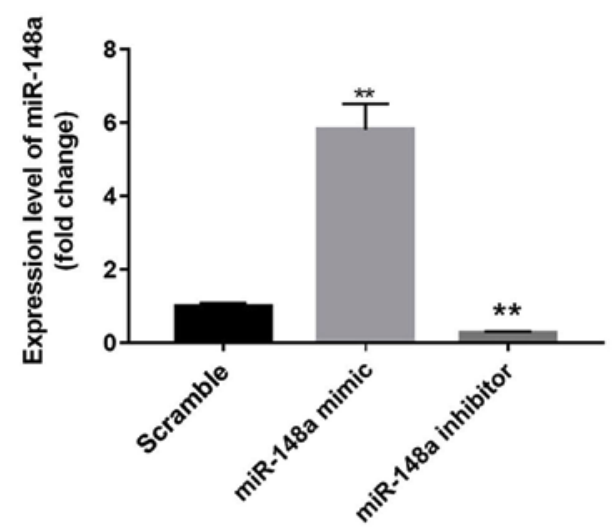

D

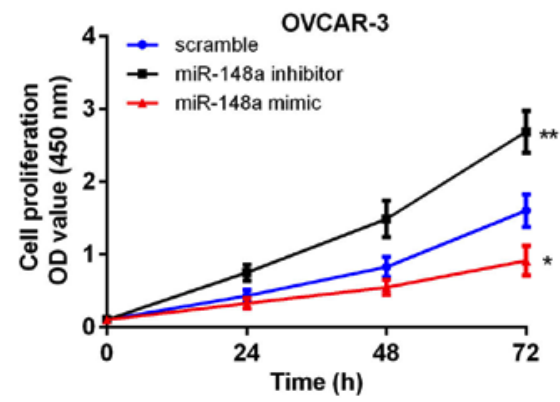

B

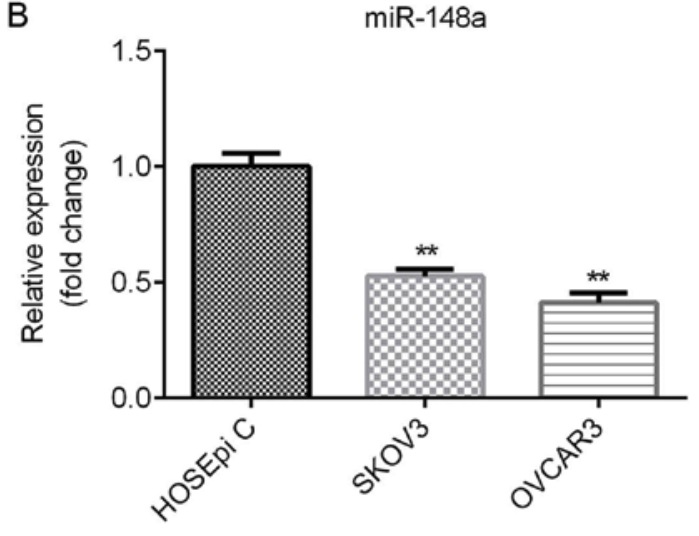

SKOV3


Figure 1. miR-148a expression is negatively associated with ovarian cancer viability. (A) miR-148a expression in ovarian cancer tissues compared with the paired adjacent normal tissues; ${ }^{* *} \mathrm{P}<0.01$. (B) Expression of miR-148a in ovarian cancer cell lines OVCAR3 and SKOV3, and normal human ovarian surface epithelial cell line HOSEpiC; ${ }^{* *} \mathrm{P}<0.01$ vs. HOSEpiC. (C) Expression of miR-148a in OVCAR3 and SKOV3 cells transfected with miR-148a mimic, inhibitor or scramble; ${ }^{* *} \mathrm{P}<0.01$ vs. scramble. (D) Cell viability in OVCAR3 and SKOV3 cells transfected with miR-148a mimic, inhibitor and scramble; ${ }^{*} \mathrm{P}<0.05$ and ${ }^{* *} \mathrm{P}<0.01$ vs. scramble. miR, microRNA; OD, optical density.

independent experiments were carried out for each assay. The differences between multiple groups was determined using one-way ANOVA with Fisher's Least Significant Difference test. Student's t-test was used to compare the difference between two groups. The clinical information in Table I was analyzed by $\chi^{2}$ test. Pearson's correlation analysis was used to analysis the correlation between miR-148a and FOXO3 gene expression. $\mathrm{P}<0.05$ was considered to indicate a statistically significant difference.

\section{Results}

miR-148a is negatively associated with ovarian cancer viability. miR-148a expression was assessed in 20 human ovarian cancer and adjacent normal tissues. As presented in Fig. 1A, the expression level of miR-148a in the cancer tissues decreased significantly compared with that in the adjacent normal tissues $(\mathrm{P}<0.01)$. Furthermore, the expression of miR-148a was monitored in ovarian cancer cell lines OVCAR3 and SKOV3, and normal human ovarian surface epithelial cell line HOSEpiC, and it was observed that the expression level of miR-148a was significantly downregulated in OVCAR3 and SKOV3 cells compared with HOSEpiC cells (Fig. 1B; P<0.01). Subsequently, OVCAR3 and SKOV3 cells were transfected with miR-148a mimic, inhibitor or scrambled control and the transfection efficiency is presented in Fig. 1C. The results of the CCK- 8 assay revealed that cell viability 
A

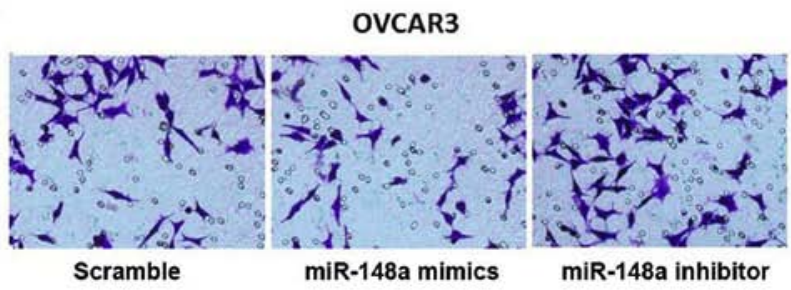

B

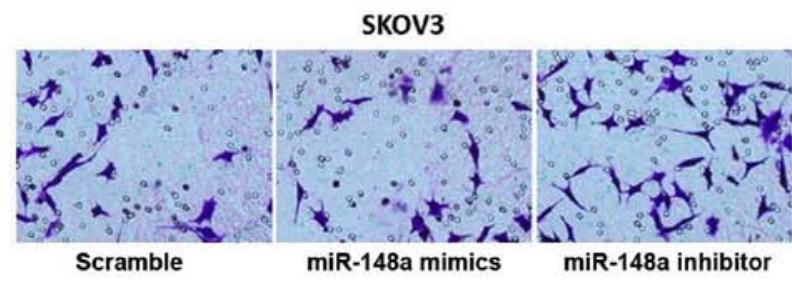

C

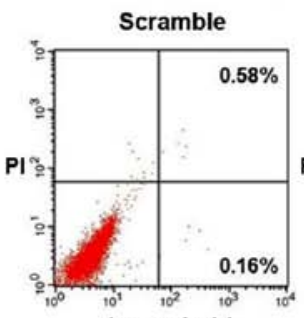

Annexin V

D

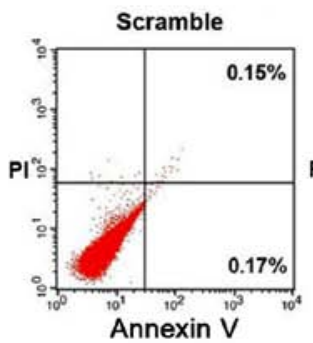

OVCAR3

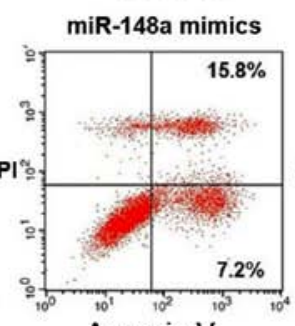

Annexin V
SKOV3

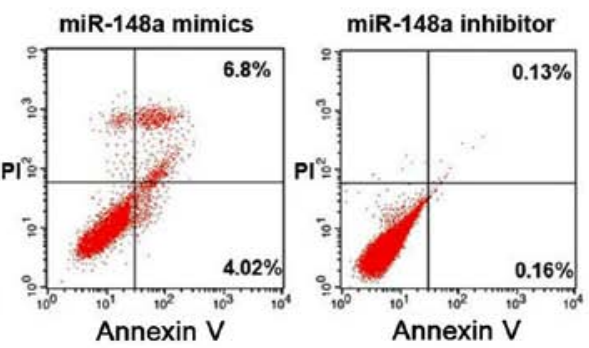

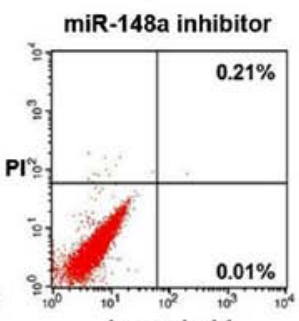

Annexin V
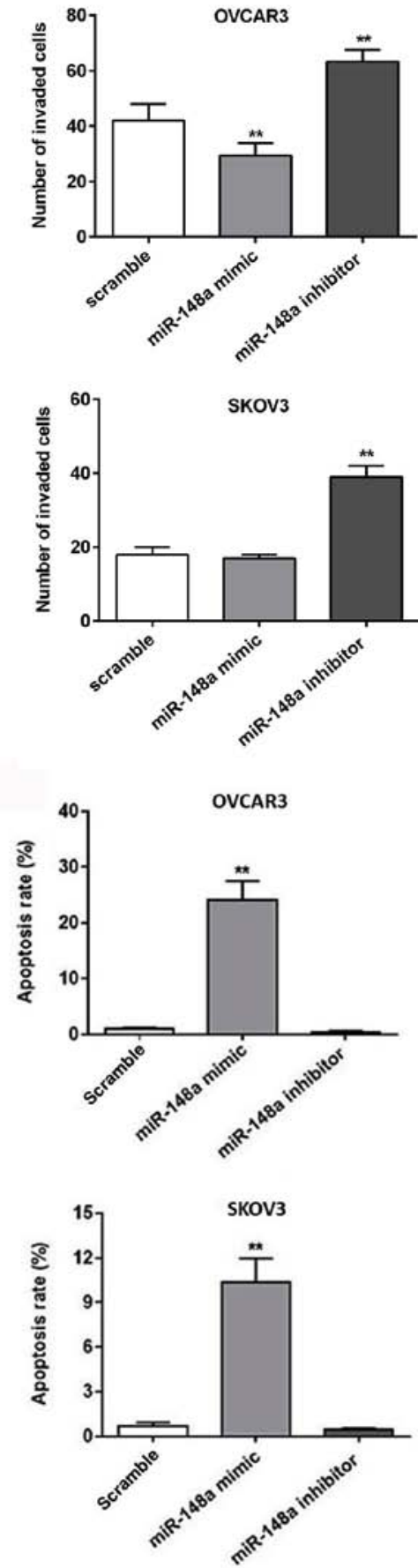

Figure 2. miR-148a decreases invasion and increases apoptosis in OVCAR3 and SKOV3 ovarian cancer cells. Invasion of (A) OVCAR3 and (B) SKOV3 cells transfected with miR-148a mimic, inhibitor or scramble. Apoptosis in (C) OVCAR3 and (D) SKOV3 cells transfected with miR-148a mimic, inhibitor or scramble; ** $\mathrm{P}<0.01$ vs. scramble; magnification $\times 200$. miR, microRNA.

was inhibited when miR-148a was overexpressed, while the miR-148a inhibitor promoted cell viability (Fig. 1D). These results suggested that miR-148a expression may be associated with reduced ovarian cancer cell growth.

miR-148a inhibits cell invasion and promotes cell apoptosis in vitro. To further investigate the anti-tumor role of miR-148a, the effects of miR-148a overexpression on cell invasion and apoptosis were determined. Transwell invasion assays showed that transfection with the miR-148a mimic suppressed the invasive ability of OVCAR3 cells to $68.8 \%$ of that of the scrambled control, but miR-148a mimic had no significant effect on the invasion of SKOV3 cells. miR-148a knockdown led to increased invasion (Fig. $2 \mathrm{~A}$ and $\mathrm{B} ; \mathrm{P}<0.01$ ). In addition, miR-148a overexpression was found to promote apoptosis of OVCAR3 and SKOV3 cells. Cells transfected with miR-148a mimic had an apoptosis rate markedly higher than the scrambled control (Fig. 2C and D; $\mathrm{P}<0.01$ ). The apoptosis rate was lower in cells transfected with the miR-148a inhibitor, but no significant differences were observed. The results indicated that miR-148a may act as a tumor suppressor for ovarian cancer by inhibiting cell invasion and promoting cell apoptosis. 
A

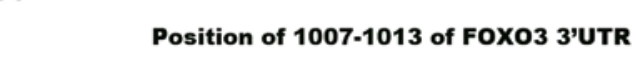

hsa-miR-148a-5p

FOX03 3'UTR mutant

B

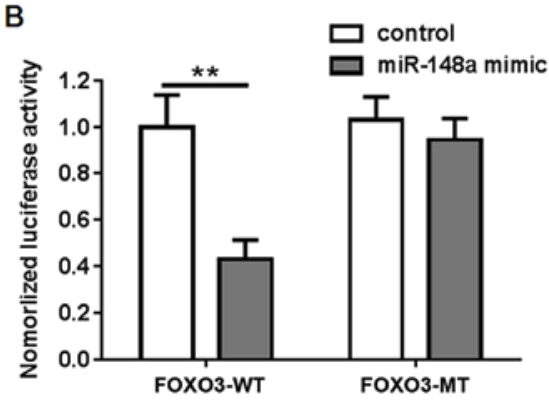

D

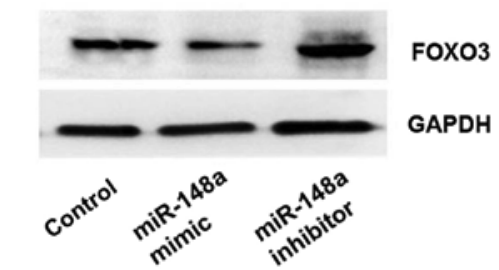

$\mathbf{F}$

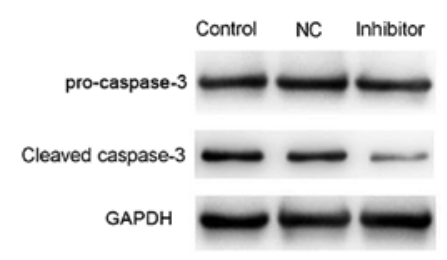

G

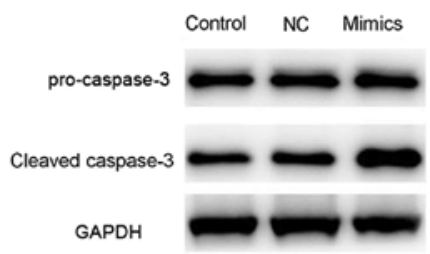

Predicted consequential pairing of target region

5'... CUUUGCAGAACAAAUGAACUUAC...

3'... UCAGCCUCACAGAGUCUUGAAA...

5'... CUUUGCAgaAcaAaUUUGAaUAC...

C

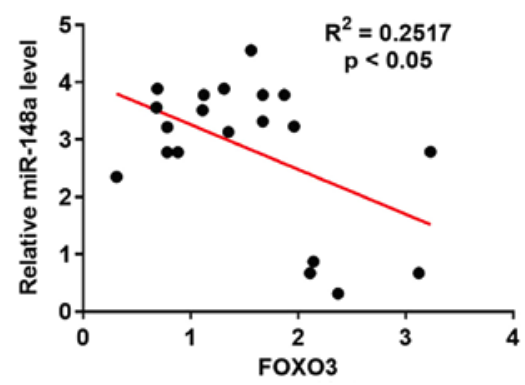

E
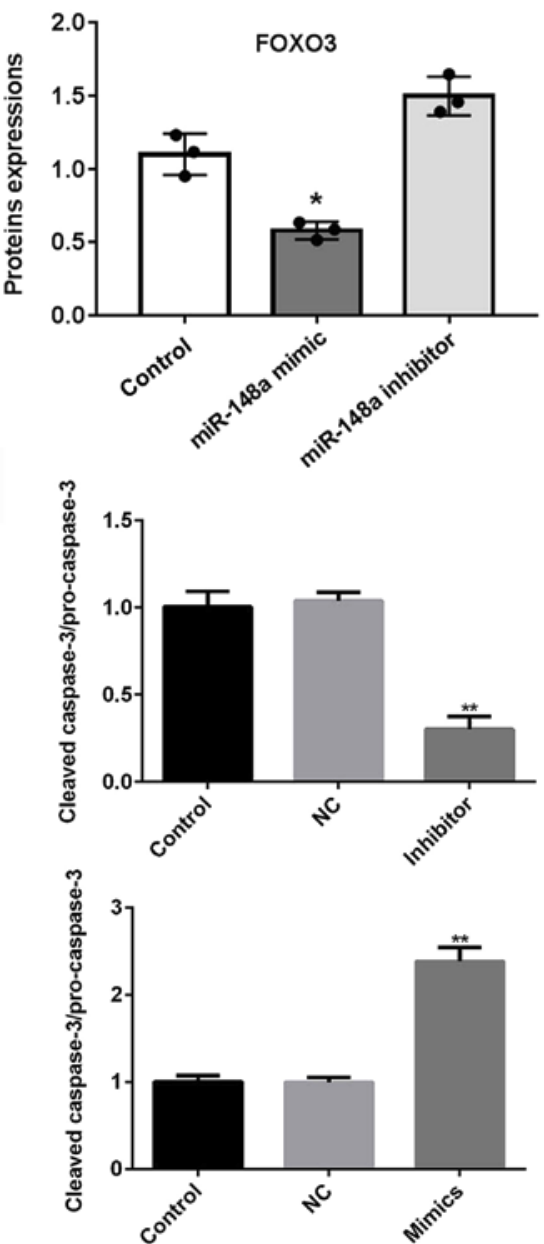

Figure 3. miR-148a targets FOXO3. (A) miR-148a sequence and the potential targeting region in the 3'UTR of FOXO3. (B) Luciferase activity in cells transfected with WT or MT FOXO3 3'UTR and miR-148a mimic; ${ }^{* *} \mathrm{P}<0.01$. (C) Correlation between the expression of miR-148a and FOXO3 mRNA in ovarian cancer tissues. Western blot (D) images and (E) quantification of FOXO3 protein expression in OVCAR3 cells transfected with miR-148a mimic, inhibitor or scramble; ${ }^{*} \mathrm{P}<0.05$ vs. control. Western blot images and quantification of cleaved caspase-3/pro-caspase-3 ratio in cells transfected with (F) miR-148a inhibitor or (G) mimic; ${ }^{* *} \mathrm{P}<0.05$ vs. control. miR, microRNA; FOXO3, forkhead box protein O3; UTR, untranslated region; WT, wild type; MT, mutant; NC, scrambled control.

miR-148a directly targets FOXO3. The TargetScan database predicted FOXO3 as a direct target of miR-148a (Fig. 3A). Based on the prediction results, miR-148a has three binding sites on the 3'UTR of FOXO3 which are 1007-1013,2087-2093 and 2262-2268. Therefore, a dual-luciferase assay was performed to verify that miR-148a targets FOXO3 3'-UTR. The FOXO3-WT or FOXO3-MT was co-transfected with the miR-148a mimic into OVCAR3 cells. The luciferase activity significantly decreased in cells co-transfected with FOXO3-WT and miR-148a mimic (Fig. 3B; P<0.01), but 
not in cells co-transfected with the mutant FOXO3-3'UTR sequence and miR-148a mimic. Furthermore, in the ovarian cancer tissues, the level of FOXO3 gene expression was negatively correlated with the expression of miR-148a (Fig. 3C). Moreover, western blotting revealed that the FOXO3 protein level was downregulated by transfection with miR-148a mimic and upregulated by transfection with miR-148a inhibitor (Fig. 3D and E). The effect of the miR-148a level on cleaved caspase-3, a cell death protease, was also investigated. As shown in Fig. 3F and G, miR-148a inhibitor decreased the expression of cleaved caspase-3 and miR-148a overexpression increased the expression of cleaved caspase-3, which may explain the pro-apoptotic role of miR-148a.

miR-148a suppresses tumor growth in nude mice. The role of miR-148a in regulating ovarian cancer growth was further studied by tumor xenograft assay. Nude mice were injected with OVCAR3 cells transfected with miR-148a mimic or inhibitor. Both the tumor size and weight were smaller in the mice injected with cells overexpressing miR-148a compared with the mice injected with normal OVCAR3 cells. Tumors derived from cells with miR-148a knockdown exhibited increased tumor growth in nude mice compared with control animals (Fig. 4). The results indicated that miR-148a may suppress tumor growth in vivo.

\section{Discussion}

miRNAs are regulators of many oncobiological processes. In the present study, miR-148a overexpression inhibited cell viability, suppressed invasion and promoted apoptosis of OVCAR3 and SKOV3 cells. miR-148a also inhibited tumor growth in vivo. The regulatory role of miR-148a may be mediated by the FOXO3 transcription factor.

miR-148a was reported to be downregulated in plasma samples of ovarian cancer patients (16). miR-148a was also discovered to be decreased in ovarian cancer tissues of $65 \%$ of patients compared with the adjacent tissue, but the difference in expression was not significant (7). Other studies also demonstrated that miR-148a is significantly downregulated in ovarian tissues compared with adjacent normal tissues $(23,24)$, which is consistent with the results of the present study. Similarly, previous studies demonstrated that miR-148a regulates growth and apoptosis of ovarian cancer cells in vitro $(23,25)$. In addition, the present study further illustrated that miR-148a reduced ovarian tumorigenesis in vivo.

Previous studies have identified several targets of miR-148a. Sphingosine-1-phosphate receptor 1 is a confirmed target of miR-148a in ovarian cancer SKOV3 cells (17). miR-148a also promotes apoptosis of OVCAR8 cells by targeting Erbb3 and MYB (26). miR-148a inhibits gastric cancer cell proliferation and invasion via inhibition of transforming growth factor- $\beta$-induced 2 (24). miR-148a was shown to promote paclitaxel-induced apoptosis of SKOV3 cells via direct inhibition of protein disulfide isomerase family A member 3 , indicating that miR-148a is associated with chemotherapy efficacy (23). FOXO3 was predicted as a target of miR-148a in the current study; however, it remains unclear whether miR-148a exerts

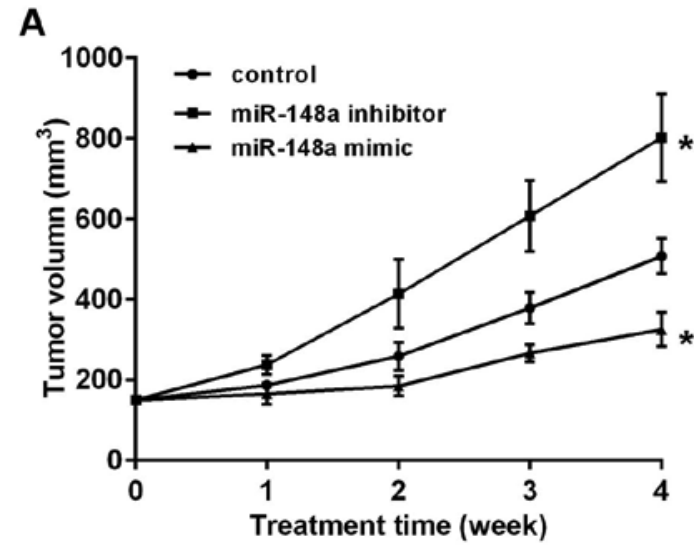

B


Figure 4. miR-148a suppresses tumor growth in a xenograft assay in nude mice injected with normal OVCAR 3 cells or OVCAR3 cells transfected with miR-148a mimic or inhibitor. (A) Tumor volume and (B) tumor weight in the nude mice at week 4. (C) Images of the primary tumors formed in the nude mice at week $4 .{ }^{*} \mathrm{P}<0.05$ vs. control. miR, microRNA.

its antitumor function in ovarian cancer cells via regulation of expression of FOXO3.

In the present study, the association between miR-148a and FOXO3 in ovarian cancer was explored. It was determined that the expression of miR-148a and FOXO3 mRNA were negatively correlated in ovarian cancer tissues and it was demonstrated that miR-148a inhibited FOXO3 expression in vitro. It is noteworthy that FOXO3 must translocate to the nucleus to initiate expression of tumor suppressing genes (27). IкB kinase (IKK) expression or phosphorylated Akt could induce nuclear exclusion of FOXO3 and contributed to cancer cell proliferation (28). Phosphorylation of Akt (29) and activation 
of IKK are commonly observed in ovarian cancer (30). This additional regulatory mechanism of FOXO3 may override the upregulation by miR-148a. Similar results were observed by Liu et al (31). That study reported that miR-498 was markedly downregulated in ovarian cancer cells and FOXO3 was a direct target of miR-498 (31). These findings suggested that the mechanisms by which FOXO3 affects tumorigenesis are complex.

The present study has limitations. First, the number of the clinical samples included in the present study was relatively small and results should be verified using a larger sample size. Second, miR-148a has three binding sites on the 3'UTR of FOXO3 which are 1007-1013, 2087-2093 and 2262-2268, as predicted by Targetscan. In the present study, only the 1007-1013 site was verified and therefore, the other two sites should be verified in future studies.

In conclusion, the present study indicated that miR-148a may act as a tumor suppressor in ovarian cancer OVCAR3 and SKOV3 cells. The suppressive effect may be due to inhibiting cell viability and invasion as well as promoting apoptosis. FOXO3 was described as a target of miR-148a and mediated the anti-tumor role. Nevertheless, further study is required to understand the complex regulatory network of miR-148a.

\section{Acknowledgments}

Not applicable.

\section{Funding}

This research was supported by the Research Project of Jiangsu Province Association of Maternal and Child Health (grant no. FYX201717) and the Research Project of Jiangsu Taizhou People's Hospital (grant nos. ZL201726 and ZL201734).

\section{Availability of data and materials}

The datasets used and/or analyzed during the current study are available from the corresponding author on reasonable request.

\section{Authors' contributions}

HQ and HG designed the study and provided funding. DZ and DY performed most of the experiments and wrote the manuscript. RG and LZ performed some of the clinical studies and analyzed the data. TG, YZ, JW and XC performed some of the cell studies.

\section{Ethics approval and consent to participate}

The study protocol was approved by the Ethics Committee Board of Taizhou People's Hospital (Taizhou, China). Informed consent was obtained from the patients.

\section{Patient consent for publication}

Not applicable.

\section{Competing interests}

The authors declare that they have no competing interests.

\section{References}

1. Chen W, Zheng R, Baade PD, Zhang S, Zeng H, Bray F, Jemal A, $\mathrm{Yu}$ XQ and He J: Cancer statistics in China, 2015. CA Cancer J Clin 66: 115-132, 2016.

2. Ozols RF: Treatment goals in ovarian cancer. Int J Gynecol Cancer 1 (15 Suppl): S3-S11, 2005.

3. Kleppe M, Amkreutz LC, Van Gorp T, Slangen BF, Kruse AJ and Kruitwagen RF: Lymph-node metastasis in stage I and II sex cord stromal and malignant germ cell tumours of the ovary: A systematic review. Gynecol Oncol 133: 124-127, 2014.

4. Calin GA, Sevignani C, Dumitru CD, Hysolp T, Noch E, Yendamuri S, Shimizu M, Rattan S, Bullrich F, Negrini M and Croce CM: Human microRNA genes are frequently located at fragile sites and genomic regions involved in cancers. Proc Natl Acad Sci USA 101: 2999-3004, 2004.

5. Hayes J, Peruzzi PP and Lawler SE: MicroRNAs in cancer: Biomarkers, functions and therapy. Trends Mol Med 20: 460-469, 2014.

6. Duan F, Liu W, Fu X, Feng Y, Dai L, Cui S and Yang Z: Evaluating the prognostic value of miR-148/152 family in cancers: Based on a systemic review of observational studies. Oncotarget 8: 77999-78010, 2017.

7. Zhou X, Zhao F, Wang ZN, Song YX, Chang H, Chiang Y and Xu HM: Altered expression of miR-152 and miR-148a in ovarian cancer is related to cell proliferation. Oncol Rep 27: 447-454, 2012.

8. Xu Q, Jiang Y, Yin Y, Li Q, He J, Jing Y, Qi YT, Xu Q, Li W, Lu B, et al: A regulatory circuit of miR-148a/152 and DNMT1 in modulating cell transformation and tumor angiogenesis through IGF-IR and IRS1. J Mol Cell Biol 5: 3-13, 2013.

9. Ma F, Feng Y, Li W, Li Z, Liu T and Li L: miR-148a suppresses estrogen-induced viability and migration of breast cancer cells via inhibition of estrogen receptor $\alpha$ expression. Exp Ther Med 13: 2515-2522, 2017.

10. Zhang H, Li Y, Huang Q, Ren X, Hu H, Sheng H and Lai M: MiR-148a promotes apoptosis by targeting Bcl-2 in colorectal cancer. Cell Death Differ 18: 1702-1710, 2011.

11. Chen Y, Song Y, Wang Z, Yue Z, Xu H, Xing C and Liu Z: Altered expression of miR-148a and miR-152 in gastrointestinal cancers and its clinical significance. J Gastrointest Surg 14: 1170-1179, 2010.

12. Murata T, Takayama K, Katayama S, Urano T, Horie-Inoue K, Ikeda K, Takahashi S, Kawazu C, Hasegawa A, Ouchi Y, et al: miR-148a is an androgen-responsive microRNA that promotes LNCaP prostate cell growth by repressing its target CAND1 expression. Prostate Cancer Prostatic Dis 13: 356-361, 2010.

13. Li L, Liu Y, Guo Y, Liu B, Zhao Y, Li P, Song F, Zheng H, Yu J, Song T, et al: Regulatory miR-148a-ACVR1/BMP circuit defines a cancer stem cell-like aggressive subtype of hepatocellular carcinoma. Hepatology 61: 574-584, 2015.

14. Han C, Zheng W, Ge M, Wang K, Xiang Y and Huang P: Downregulation of cyclin-dependent kinase 8 by microRNA-148a suppresses proliferation and invasiveness of papillary thyroid carcinomas. Am J Cancer Res 7: 2081-2090, 2017.

15. Kim EA, Kim TG, Sung EG, Song IH, Kim JY, Doh KO and Lee TJ: miR-148a increases the sensitivity to cisplatin by targeting Rab14 in renal cancer cells. Int J Oncol 50: 984-992, 2017.

16. Gong L, Wang C, Gao Y and Wang J: Decreased expression of microRNA-148a predicts poor prognosis in ovarian cancer and associates with tumor growth and metastasis. Biomed Pharmacother 83: 58-63, 2016.

17. Wen Z, Zhao S, Liu S, Liu Y, Li X and Li S: MicroRNA-148a inhibits migration and invasion of ovarian cancer cells via targeting sphingosine-1-phosphate receptor 1. Mol Med Rep 12: 3775-3780, 2015.

18. Kaestner KH, Knochel W and Martinez DE: Unified nomenclature for the winged helix/forkhead transcription factors. Genes Dev 14: 142-146, 2000.

19. Fu Z and Tindall DJ: FOXOs, cancer and regulation of apoptosis. Oncogene 27: 2312-2319, 2008.

20. Kurman RJ, Carcangiu ML, Herrington CS and Young RHE: WHO Classification of Tumours of Female Reproductive Organs. 4th edition. IARC, Lyon, 2014.

21. Paik ES, Lee YY, Lee EJ, Choi CH, Kim TJ, Lee JW, Bae DS and Kim BG: Survival analysis of revised 2013 FIGO staging classification of epithelial ovarian cancer and comparison with previous FIGO staging classification. Obstet Gynecol Sci 58: 124-134, 2015. 
22. Livak KJ and Schmittgen TD: Analysis of relative gene expression data using real-time quantitative PCR and the 2(-Delta Delta C(T)) method. Methods 25: 402-408, 2001.

23. Zhao S, Wen Z, Liu S, Liu Y, Li X, Ge Y and Li S: MicroRNA-148a inhibits the proliferation and promotes the paclitaxel-induced apoptosis of ovarian cancer cells by targeting PDIA3. Mol Med Rep 12: 3923-3929, 2015.

24. Zhou M, Su Z, Zhang S, Zhuang L, Xie Y and Li X: Suppressive role of microRNA-148a in cell proliferation and invasion in ovarian cancer through targeting transforming growth factor- $\beta$-induced 2. Oncol Res 24: 353-360, 2016.

25. Wang W, Dong J, Wang M, Yao S, Tian X, Cui X, Fu S and Zhang S: miR-148a-3p suppresses epithelial ovarian cancer progression primarily by targeting c-Met. Oncol Lett 15 . 6131-6136, 2018.

26. Ghoshchoudhury T, Xiao W, Gunaratne PH and Anderson ML: Loss of miR-148a/b expression promotes ovarian cancer by targeting Erbb3 and MYB. Gynecol Oncol 125 (Suppl 1): S98, 2012

27. Carbajo-Pescador S, Mauriz JL, García-Palomo A and González-Gallego J: FoxO proteins: Regulation and molecular targets in liver cancer. Curr Med Chem 21: 1231-1246, 2014.
28. Hu MC, Lee DF, Xia W, Golfman LS, Ou-Yang F, Yang JY, Zou Y, Bao S, Hanada N, Saso H, et al: IkappaB kinase promotes tumorigenesis through inhibition of forkhead FOXO3a. Cell 117: 225-237, 2004

29. Dobbin ZC and Landen $\mathrm{CN}$ : The importance of the $\mathrm{PI} 3 \mathrm{~K} / \mathrm{AKT} / \mathrm{MTOR}$ pathway in the progression of ovarian cancer. Int J Mol Sci 14: 8213-8227, 2013.

30. Ataiekachoie P, Badar S, Morris DL and Pourgholami MH: Minocycline targets the NF- $\kappa \mathrm{B}$ nexus through suppression of TGF- $31-T A K 1-I \kappa B$ signaling in ovarian cancer. Mol Cancer Res 11: 1279-1291, 2013.

31. Liu R, Liu F, Li L, Sun M and Chen K: MiR-498 regulated FOXO3 expression and inhibited the proliferation of human ovarian cancer cells. Biomed Pharmacother 72: 52-57, 2015.

(c) (i) () $($ This work is licensed under a Creative Commons EY No ND Attribution-NonCommercial-NoDerivatives 4.0 International (CC BY-NC-ND 4.0) License. 